\title{
Baicalein Potently Inhibits Rho Kinase Activity and Suppresses Actin Stress Fiber Formation in Angiotensin II-Stimulated H9c2 Cells
}

\author{
Kwang-Seok Oh, Byung Koo Oh, Cheon Ho Park, Jihye Mun, Suk Hyun Won, and \\ Byung Ho Lee* \\ Bio-Organic Science Division, Korea Research Institute of Chemical Technology; Daejeon 305-343, Republic of \\ Korea. Received February 6, 2012; accepted May 11, 2012
}

Baicalein is a flavonoid (5,6,7-trihydroxy-2-phenyl-4H-1-benzopyran4-one) and an active principle in Scutellaria baicalensis. The present study was performed to investigate the mechanisms underlying the antifibrotic effects of baicalein with a focus on Rho kinase (ROCK) inhibition. The effect of baicalein on ROCK activity was analyzed using an immobilized metal affinity for phosphochemicals (IMAP)-based time-resolved fluorescence resonance energy transfer (TR-FRET) assay. The underlying mechanisms of baicalein were examined using angiotensin II-stimulated H9c2 cells. Rho kinase (ROCK1 and ROCK2) studies using IMAPTR-FRET showed that baicalein possesses potent ROCK inhibitory activity with $\mathrm{IC}_{50}$ values of 6.55 and $2.82 \mu \mathrm{M}$, respectively. Pretreatment with baicalein (for $2 \mathrm{~h}$ ) concentration-dependently decreased the angiotensin II-induced phosphorylation of myosin phosphatase (MYPT) and myosin light chain (MLC). Furthermore, baicalein also concentration-dependently suppressed actin stress fiber formation in angiotensin II-stimulated H9c2 cells. These results suggest that baicalein potently inhibits ROCK and that by so doing it modulates actin stress fiber formation. These anti-fibrotic effects of baicalein explain, at least in part, its pharmacology and mode of action.

Key words baicalein; Rho kinase; angiotensin II; actin stress fiber; H9c2 cell

Rho kinase (ROCK), a downstream effecter of Rho, is a serine-threonine kinase activated by interaction with the active guanosine 5'-triphosphate (GTP)-bound form of Rho A. ROCK participates in many vascular functions, such as, actin stress fiber formation, ${ }^{1)}$ smooth muscle contraction ${ }^{2)}$ and membrane ruffling. ${ }^{3)}$ Although the roles of the two isoforms, ROCK1 and ROCK2, are not entirely clear, ROCK1 mRNA is preferentially expressed in lung, liver, spleen, kidney, and testis, whereas ROCK2 mRNA shows highest expression in the heart and brain., ${ }^{4)}$ Excessive activation of ROCK has been demonstrated in several cardiovascular diseases, such as, coronary artery disease, restenosis, pulmonary hypertension, stroke, and heart failure. ${ }^{6,7)}$ As such, Rho kinase inhibitors have been considered to provide a pharmacological strategy for preventing and treating cardiovascular diseases. ${ }^{8)}$

Herbal medicines have been empirically used as therapeutic alternatives to treat those suffering from cardiovascular disorders and many studies have been undertaken to provide scientific proof that justifies their medicinal use for the treatment of cardiovascular diseases. ${ }^{9}{ }^{910}$ Scutellaria baicalensis is one of the most versatile oriental herbal drugs, and has been used clinically to treat or prevent cardiovascular conditions, such as, hypertension, atherosclerosis. ${ }^{11,12}$ It contains a variety of flavones, amino acids, and essentials oils, and over 30 flavonoids, including baicalein, baicalin and wogonin, have been found in dried root preparations. ${ }^{13)}$ Baicalein is one of the main active ingredients in Scutellaria baicalensis ${ }^{14)}$ (Fig. 1), and has been reported to possess diverse biological activities, such as, to protect rat cardiomyocytes against hypoxia, ${ }^{15)}$ to scavenging ROS generation in cardiomyocytes, ${ }^{16)}$ and to improve cardiac contractile function in endotoxemic rats. ${ }^{17)}$

Recently, it was suggested that baicalein inhibits cardiac fibrosis in spontaneously hypertensive rats, ${ }^{18)}$ but the molecular processes responsible for the anti-fibrotic effects of baicalein

The authors declare no conflict of interest. have not been determined. In preliminary studies, we found that baicalein inhibited the activation of ROCK. Based on these considerations, we have presumed that baicalein may be potential ROCK inhibitor, which is critically involved in the suppression of stress fiber organization in cardiomyocytes against various pathological stimuli. Accordingly, the present study was undertaken to assess the effect of baicalein on ROCK activity and to explore the mechanism of baicalein in rat heart derived $\mathrm{H} 9 \mathrm{c} 2$ cells stimulated with angiotensin II.

\section{MATERIALS AND METHODS}

Chemicals and Reagents Y-27632 [4-(1-Aminoethyl)- $N$ (4-pyridyl)cyclohexanecarboxamide dihydrochloride monohydrate], a Rho kinase selective reference compound, ${ }^{19)}$ and baicalein (5,6,7-trihydroxyflavone) were purchased from Sigma-Aldrich (St. Louis, MO, U.S.A.). Human recombinant ROCK1 and ROCK2 were purchased from Upstate (Millipore Co., Billerica, MA, U.S.A.). The fluorescent peptide substrate (FAM-S6 ribosomal protein-derived peptide) and an immobilized metal affinity for phosphochemicals (IMAP)based time-resolved fluorescence resonance energy transfer (TR-FRET) Screening Express Kit were obtained from MDS Analytical Technologies (Sunnyvale, CA, U.S.A.). Dulbecco's modified Eagle's medium (DMEM), fetal bovine serum, and antibiotics were purchased from GIBCO BRL (Grand Islang, NY, U.S.A.). Anti-myosin phosphatase (MYPT1), anti-phosphorylated MYPT1 (anti-p-MYPT1), anti-myosin light chain

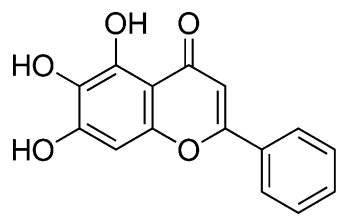

Fig. 1. The Chemical Structure of Baicalein 
2 (anti-MLCP2), and anti-phosphorylated MLCP2 (anti-pMLCP2) rabbit polyclonal antibodies were purchased from Cell Signaling Technology (Danvers, MA, U.S.A.). Alexa fluor 586 phalloidin (an F-actin probe) was purchased from Invitrogen (Carlsbad, CA, U.S.A.).

ROCK-TR-FRET Assay Assays were performed in 384well white flat-bottom plates (Corning Life Sciences, Lowell, MA, U.S.A.). A ROCK assay was performed using $0.4 \mu \mathrm{g} / \mathrm{mL}$ ROCK 1 or $0.1 \mu \mathrm{g} / \mathrm{mL}$ ROCK 2 in kinase reaction buffer $(10 \mathrm{~mm}$ Tris- $\mathrm{HCl}, \mathrm{pH} 7.2,10 \mathrm{~mm} \mathrm{MgCl}_{2}, 0.05 \% \mathrm{NaN}_{3}$ ), containing $0.01 \%$ Tween-20 and $1 \mathrm{~mm}$ dithiothreitol. Fluorescein-tagged S6 ribosomal protein-derived substrate (5FAM-AKRRRLSSL$\mathrm{RA}-\mathrm{COOH}$ ) and ATP were then added at final concentrations of $1 \mu \mathrm{M}$ or $3 \mu \mathrm{M}$, respectively. The total reaction volume was $20 \mu \mathrm{L}$ and baicalein $(0.1-100 \mu \mathrm{M})$ was preincubated with ROCK enzyme for $10 \mathrm{~min}$ before adding the S6 ribosomal protein-derived substrates substrate and ATP. Kinase reactions were conducted for $45 \mathrm{~min}$ at room temperature in white standard 384-well plates and then $60 \mu \mathrm{L}$ of detection mixture (1:600 dilution of IMAP binding reagent and 1:400 dilution of Terbium donor supplied by MDS Analytical Technologies) was added to kinase reaction plates $3 \mathrm{~h}$ before reading the plates. The TR-FRET counts were measured using an Envision (PerkinElmer Oy, Turku, Finland) multilabel counter with a TR-FRET option. The instrument settings used were $340 \mathrm{~nm}$ for excitation and $520 \mathrm{~nm}$ and $495 \mathrm{~nm}$ for emission with a $100 \mu$ s delay time. Measured TR-FRET counts were used to calculate percent inhibitions and $\mathrm{IC}_{50}$ values. In kinase selectivity assay, myosin light-chain kinase (MLCK) and protein kinase $\mathrm{C} \alpha(\mathrm{PKC} \alpha)$ assays were performed by kinase profiler service (Millipore, Billerica, MA, U.S.A.).

Cell Culture Rat heart-derived H9c2 cells were purchased from the American Type Culture Collection (ATCC, Rockville, MD, U.S.A.) and maintained at $1 \times 10^{6}$ cells $/ \mathrm{mL}$ in DMEM supplemented with 10\% FBS, penicillin G (100 IU/ $\mathrm{mL})$, streptomycin $(100 \mu \mathrm{g} / \mathrm{mL})$, and L-glutamine $(2 \mathrm{~mm})$ in a $37^{\circ} \mathrm{C}$ humidified atmosphere containing $5 \% \mathrm{CO}_{2}$ and $95 \%$ air. Cells were then starved in serum free media for $3 \mathrm{~h}$ and stimulated with 0.1 or $0.3 \mu \mathrm{m}$ angiotensin II in the presence or absence of baicalein $(1-30 \mu \mathrm{M})$ for the indicated times. Stock solutions of baicalein were prepared in dimethyl sulfoxide (DMSO), and the maximum concentration of DMSO in experimental media was $0.1 \%$.

3-(4,5-Dimethylthiazol-2-yl)-2,5-diphenyltetrazolium Bromide (MTT) Assay for Cell Viability H9c2 cells $\left(7 \times 10^{4}\right.$ cells/well in 24 -well plates $)$ were incubated with various concentrations of baicalein $(1-30 \mu \mathrm{M})$ for $2 \mathrm{~h}$. MTT $(50 \mu \mathrm{g} /$ $\mathrm{mL}$ ) was then added to $1 \mathrm{~mL}$ of cell suspension and incubated for $1 \mathrm{~h}$, and the formazan so formed was dissolved in DMSO. Optical densities were measured using a microplate reader (Envision, PerkinElmer Oy, Turku, Finland) at $590 \mathrm{~nm}$. The optical density of the formazan formed in medium alone was taken as $100 \%$ viability.

Immunoblotting for MYPT1 and MLC2 H9c2 cells were treated with or without baicalein $(1-10 \mu \mathrm{M})$ for $2 \mathrm{~h}$ before angiotensin II stimulation. Cells were then stimulated with $0.1 \mu \mathrm{M}$ angiotensin II for $15 \mathrm{~min}$ to determine levels of myosin phosphatase 1 (MYPT1), myosin light chain 2 (MLC2), phosphorylated MYPT1 (p-MYPT1), and phosphorylated MLC2 (p-MLC2). Angiotensin II-stimulation was stopped by adding ice-cold phosphate-buffered saline. The cells were then lysed for immunoblotting. Equal amounts of extracted proteins were separated on $12 \%$ or $8 \%$ SDS-polyacrylamide gels, transferred to nitrocellulose membranes. Blots were probed with rabbit polyclonal antibodies against MYPT1, p-MYPT1, MLC2, and p-MLC2. Proteins transferred to membranes were detected using the LumiGLO kit (New England Biolabs, Ipswich, MA, U.S.A.). All antibodies were purchased from Cell Signaling (Danvers, MA, U.S.A.) and used at a dilution of $1: 1000$. The results of Western blots were quantified by means of Scion Image (version 4.02 beta; Scion Corporation, Frederick, MD, U.S.A.).

Immunofluorescent Staining for F-Actin Stress Fiber Formation For immunofluorescent staining, H9c2 cells were plated on a chamber slide (Thermofisher, Rochester, NY, U.S.A.) at $10^{5}$ cells $/ \mathrm{mL}$. After preincubation with or without baicalein $(1-10 \mu \mathrm{M})$ for $1 \mathrm{~h}$, they were treated with angiotensin II $(0.3 \mu \mathrm{M})$ for $2 \mathrm{~h}$, fixed with $4 \%$ paraformaldehyde for $20 \mathrm{~min}$, incubated with $0.5 \%$ Triton $\mathrm{X}-100$ for $5 \mathrm{~min}$ at $-20^{\circ} \mathrm{C}$ in a freezer, and then blocked with $1 \%$ bovine serum albumin for $30 \mathrm{~min}$. Cells were then probed with Alexa fluor 586 phalloidin (Invitrogen, Carlsbad, CA, U.S.A., diluted 1:1000) for $30 \mathrm{~min}$ at room temperature in the dark, washed with phosphate buffered saline three times, and stained with Hoechst 33342 dye for $2 \mathrm{~min}$. Fluorescent images were obtained under a fluorescence microscope at $400 \times$ magnification (Nikon, Tokyo).

Statistical Analysis All values are expressed as means S.D. Data were analyzed by one-way analysis of variance (ANOVA), followed by Dunnett's test for multiple comparisons (Sigma Stat, Jandel Co., San Rafael, CA, U.S.A.). Percent inhibition was calculated using; $\left(\left[\mathrm{Mean}_{\mathrm{s}}-\mathrm{Mean}_{\mathrm{c}-}\right] /\left[\mathrm{Mean}_{\mathrm{c}+}-\right.\right.$ Mean $\left.\left._{c-}\right]\right) \times 100$. Mean $_{\mathrm{c}+}, \mathrm{Mean}_{\mathrm{c}-}$ and $\mathrm{Mean}_{\mathrm{s}}$ are the means of TR-TRET counts of the positive control, negative control, and test samples, respectively. Concentration-response curves were analyzed by nonlinear regression using PRISM version 3.0 (GraphPad Software Inc., La Jolla, CA, U.S.A.), and the $\mathrm{IC}_{50}$ value of baicalein (the concentration required to reduce the TR-FRET count to $50 \%$ of the positive control) was calculated. Statistical significance was accepted for $p$ values of $<0.05$.

\section{RESULTS}

Inhibitory Effects of Baicalein on ROCK Activities The inhibitory effects of baicalein on the activities of ROCK1 and ROCK 2 were evaluated using a kinase assay based on IMAP-TR-FRET, which involves specific interactions between proprietary nanoparticles and covalent phosphorylated moieties. Phosphosubstrate-nanoparticle binding events can be directly quantified and expressed as TR-FRET counts, which reflect the amount of ROCK-induced phosphorylation of a substrate. The assay conditions of ROCK1- and ROCK2-IMAPTR-FRET assays were verified using Y27632 as a reference compound ( $\mathrm{IC}_{50}$ values: $0.15,0.23 \mu \mathrm{M}$, respectively), which agreed with the $\mathrm{IC}_{50}$ values obtained using a radio-isotope based kinase assay $(0.22,0.30 \mu \mathrm{M}){ }^{19)}$ As shown in Fig. 2A, baicalein inhibited the ROCK1 and ROCK2-induced TR-FRET counts in a concentration-dependant manner, and provided $\mathrm{IC}_{50}$ values of 6.55 and $2.82 \mu \mathrm{M}$, respectively.

In inhibition studies, double reciprocal plots of $1 / v$ versus $1 /[\mathrm{ATP}]$ were performed using various concentration of ATP 
A

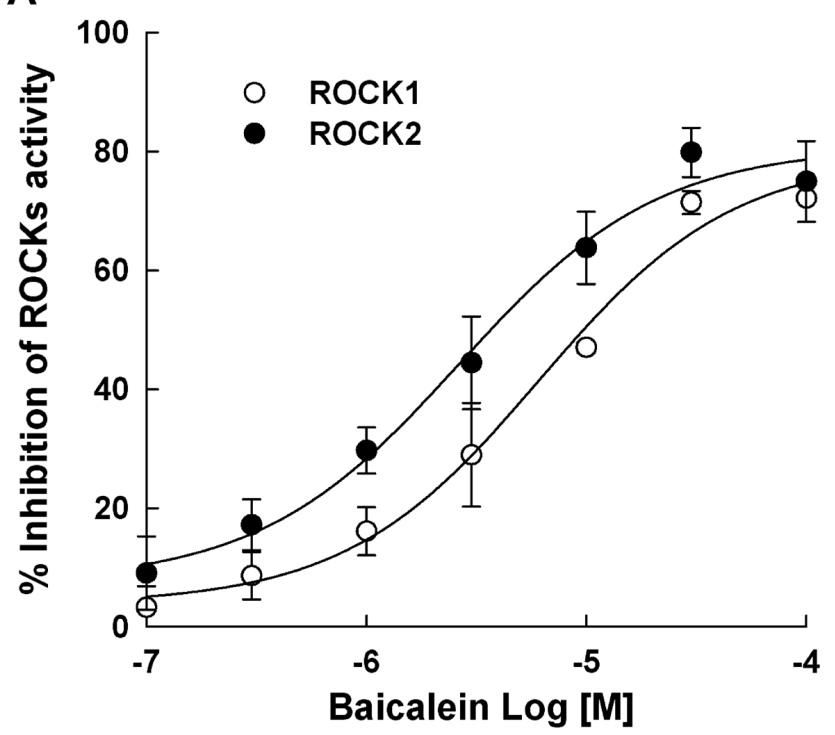

B

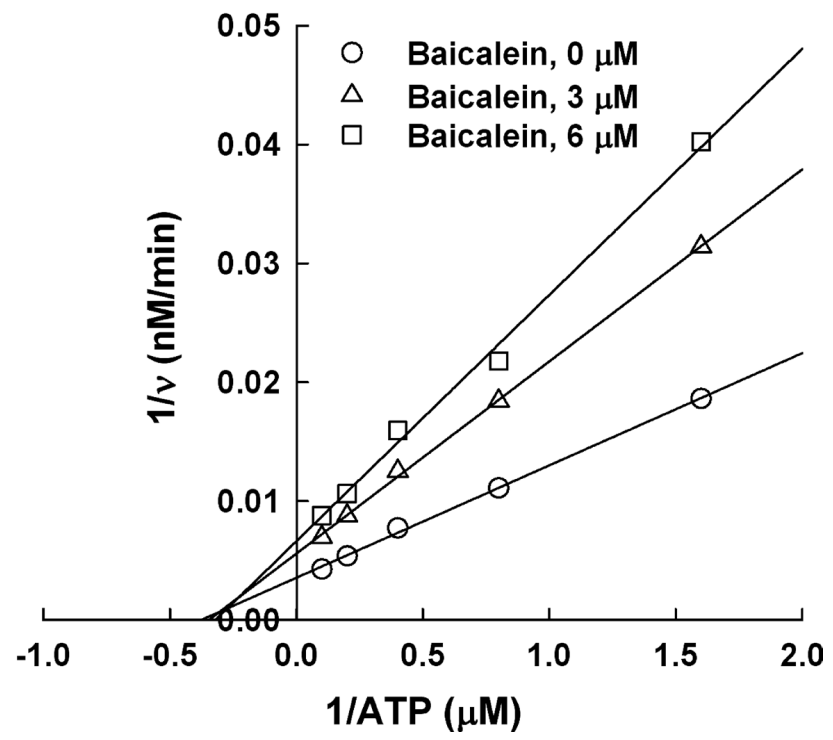

Fig. 2. (A) Inhibitory Effects of Baicalein on the Activities of ROCK1 and ROCK2, and (B) the Mode of Action of Baicalein

Rho kinase activity was determined using an IMAP-TR-FRET assay as described in Materials and Methods. Y27632 was used as a reference compound for the ROCK kinase assay. Percent inhibitions were calculated using; ([Mean ${ }_{\mathrm{s}}-$

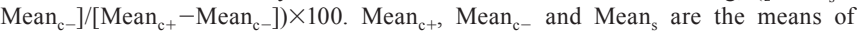
TR-TRET counts of the positive control, negative control, and test samples, respectively. Double reciprocal plots of $1 / v$ versus $1 /[\mathrm{ATP}]$ were made using differen ATP concentrations $(0.625-10 \mu \mathrm{M})$, ROCK $2(0.1 \mu \mathrm{g} / \mathrm{mL})$, and S6 ribosomal proteinderived substrate $(1 \mu \mathrm{M})$ in the absence or presence of increasing concentrations of baicalein. The initial rate $v$ was estimated to be the amount of phospho-substrate transferred during the reaction period (n $/ / \mathrm{min})$. Experiments were performed at least three times.

(0.625-10 $\mu \mathrm{M})$, ROCK2 $(0.1 \mu \mathrm{g} / \mathrm{mL})$, and S6 ribosomal proteinderived substrate $(1 \mu \mathrm{M})$ in the presence or absence of increasing concentrations of baicalein. The initial rate $v$ was defined as rate of phospho-substrate transfer (nm/min). As shown in Fig. 2B, baicalein behaved as an ATP-noncompetitive inhibitor of ROCK2.

The Cytotoxic Effect of Baicalein on H9c2 Cells To investigate the effect of baicalein on cell viability, cells were exposed to various concentrations of baicalein $(1-30 \mu \mathrm{M})$ for

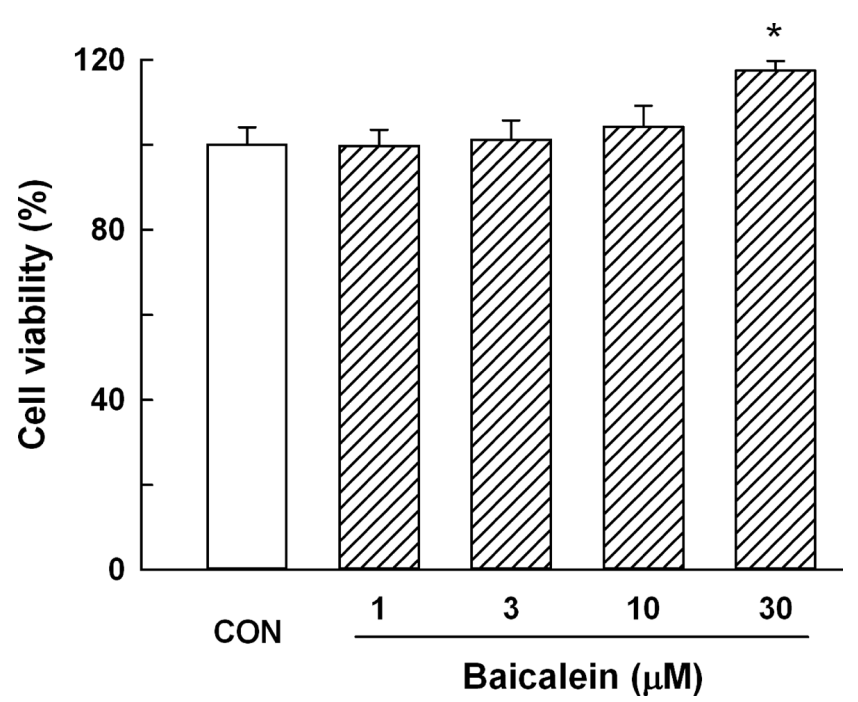

Fig. 3. The Effects of Baicalein on H9c2 Cell Viability

Cells were treated with various concentrations of baicalein for $2 \mathrm{~h}$ and cell viabilities were measured using an MTT assay. Results are presented as means \pm S.D. of experiments performed in triplicate. ${ }^{*} p<0.05$; significantly different from the control group (CON)
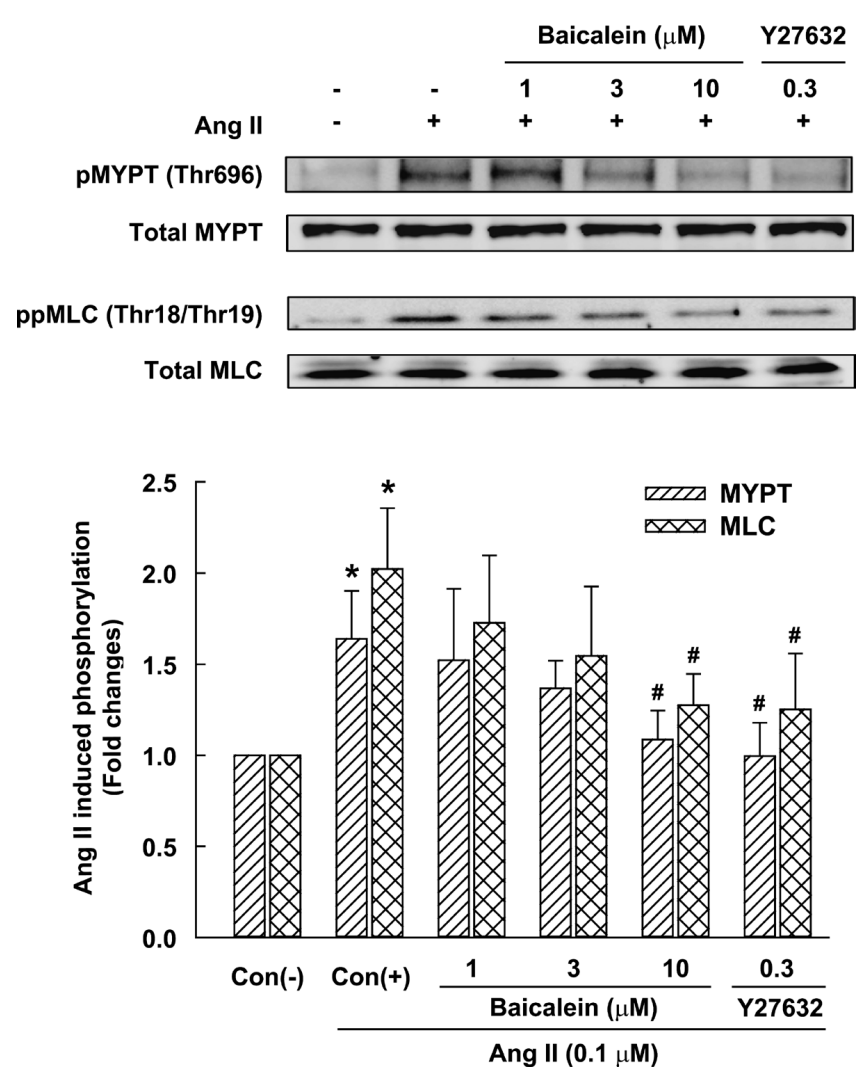

Fig. 4. Inhibition of the Angiotensin II-Induced Phosphorylations of MYPT and MLC at Various Baicalein Concentrations

Cells were pretreated for $2 \mathrm{~h}$ with or without baicalein at the indicated concentrations and then stimulated with angiotensin II $(0.1 \mu \mathrm{M})$. The levels of phosphorylated MYPT and MLC in total extracts were determined by immunoblotting. Total MYPT and MLC were used as protein-loading controls for each lane. Representative immunoblots from three experiments and densitometric evaluation are demonstrated. ${ }^{*} p<0.05$, significantly different from control cell $(-),{ }^{*} p<0.05$, significantly different from control cell $(+)$ stimulated with angiotensin II $(0.1 \mu \mathrm{M})$.

$2 \mathrm{~h}$. Baicalein did not exhibit any obvious cytotoxicity at concentrations up to $30 \mu \mathrm{M}$ (Fig. 3). The maximum concentration 


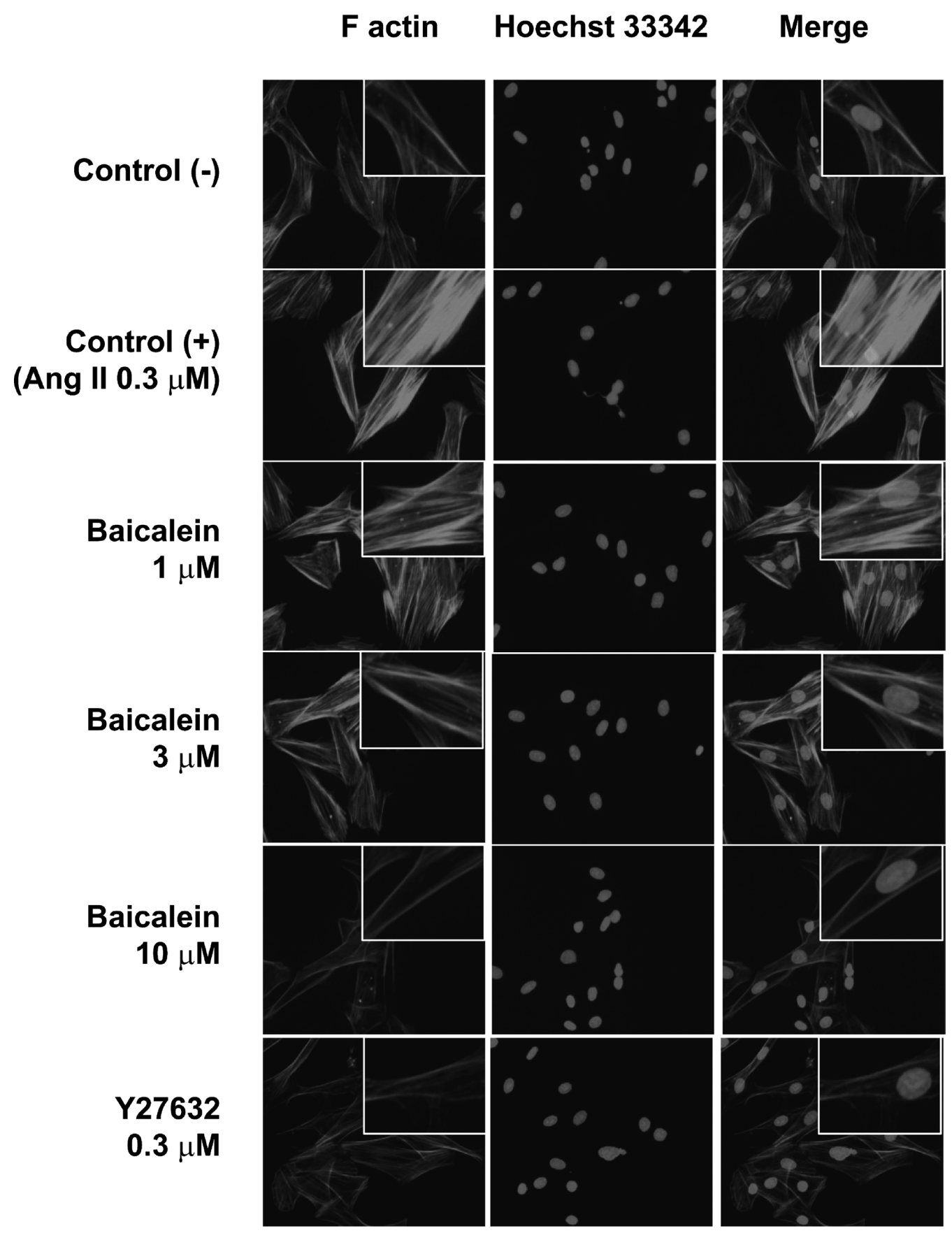

Fig. 5. Immunofluorescent Staining for Actin Stress Fiber Formation in H9c2 Cells

Cells were pretreated with baicalein at the indicated concentrations for $2 \mathrm{~h}$, and then stimulated with angiotensin II $(0.3 \mu \mathrm{M})$ for $1 \mathrm{~h}$. Actin stress fiber formation was visualized by Alexa fluor 586 phalloidin staining. Same fields were counter stained with Hoechst 33342 dye to locate nuclei. The photograph shown is representative of two independent experiments.

of baicalein was limited to $10 \mu \mathrm{M}$ for the mechanism studies because cell viability significantly increased at a concentration of $30 \mu \mathrm{M}$.

Effects of Baicalein on MYPT and MLC Phosphorylation in Activated H9c2 Cells To assess the effects of baicalein on the phosphorylations of MYPT and MLC via ROCK activation, we prepared extracts of $\mathrm{H} 9 \mathrm{c} 2$ cells activated with angiotensin II and determined phosphorylated MYPT and MLC levels by immunoblotting. As shown in Fig. 4, treatment with $0.1 \mu \mathrm{M}$ angiotensin II for $15 \mathrm{~min}$ caused the phosphorylations of MYPT and MLC in H9c2 cells and these angiotensin II-induced phosphorylations were significantly decreased by pretreatment with baicalein $(10 \mu \mathrm{M})$. In the same experiment, the phosphorylation of MYPT and MLC were significantly inhibited by pretreatment with Y-27632 $(0.3 \mu \mathrm{M})$, a Rho kinase selective reference compound.

Effects of Baicalein on Actin Stress Fiber Formation in Activated H9c2 Cells To evaluate the cellular effect of baicalein, we used an actin stress fiber formation assay. It is well known that actin stress fiber formation is induced by the activation of ROCK in several types of cells and tissues. ${ }^{20)}$ As shown in Fig. 5, treatment with angiotensin II $(0.3 \mu \mathrm{M})$ alone (Control $(+))$ increased actin stress fiber formation and this was suppressed by baicalein $(10 \mu \mathrm{M})$ pretreatment. This 


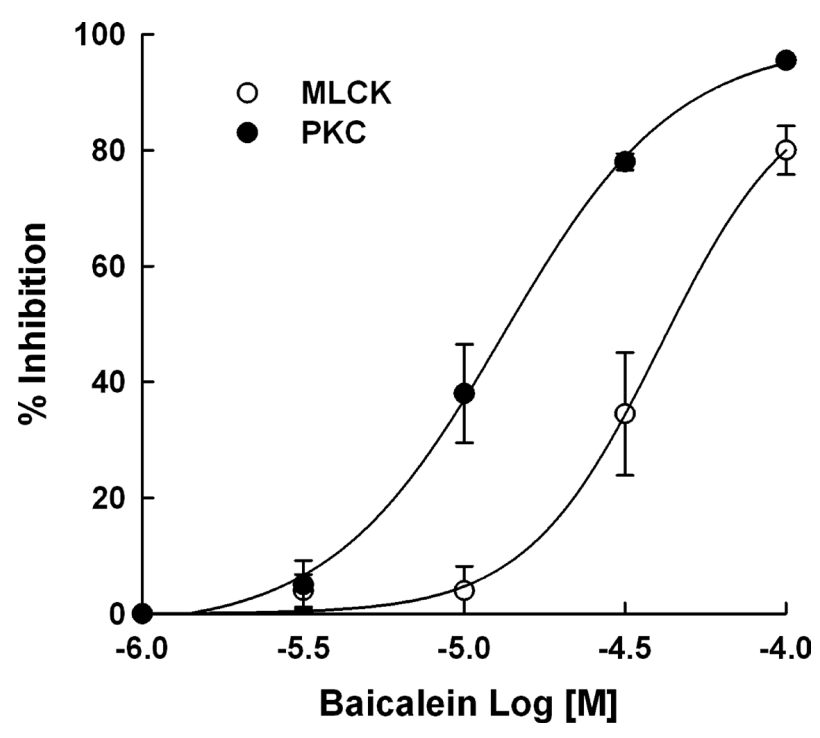

Fig. 6. Inhibitory Effects of Baicalein on the Activities of MLCK and PKC

Percent inhibitions were calculated using; ([Mean $\left.{ }_{\mathrm{s}}-\mathrm{Mean}_{\mathrm{c}-}\right] /\left[\mathrm{Mean}_{\mathrm{c}+}-\right.$ Mean $\left.\left._{\mathrm{c}-}\right]\right) \times 100$. Mean $\mathrm{c}$, Mean $_{\mathrm{c}-}$ and Mean $\mathrm{s}$ are the means of TR-TRET counts of the positive control, negative control, and test samples, respectively.

suppressive effect on actin stress fiber formation was also observed for Y27632 at $0.3 \mu \mathrm{M}$.

Inhibitory Effects of Baicalein on MLCK and PKC Activities To investigate whether decreases in phosphorylation of MLC2 are via other pathways not related Rho kinases inhibition, we measured the inhibitory effects of baicalein for MLCK and PKC $\alpha$. As shown in Fig. 6, baicalein inhibited the MLCK and PKC $\alpha$ activities in a concentration-dependant manner, and provided $\mathrm{IC}_{50}$ values of 43.4 and $15.8 \mu \mathrm{M}$, respectively.

\section{DISCUSSION}

The purpose of the present study was to assess the effect of baicalein on ROCK activities and to explore the mechanism underlying this inhibition by baicalein. Recently, we devised a high throughput screening (HTS) method to screen for ROCK1 and ROCK2 inhibitors using the IMAP-TR-FRET system. During our efforts to identify novel ROCK inhibitors from natural sources using a ROCK-TR-FRET assay, we found that baicalein inhibited the activations of ROCK1 and ROCK2 with $\mathrm{IC}_{50}$ values of 6.55 and $2.82 \mu \mathrm{M}$, respectively. Furthermore, baicalein was recently reported to inhibit cardiac fibrosis spontaneously in hypertensive rats. ${ }^{18)}$ Based on these considerations, we hypothesized that the anti-fibrotic effect of baicalein is due to its direct inhibition of ROCK.

Targeting of the Rho/ROCK pathway is considered a promising anti-fibrotic strategy in the context of preventing and/or treating injuries to the heart and lungs. ${ }^{21)}$ In addition, ROCK inhibitors may offer an attractive means of diminishing enhanced ROCK activity to decrease hypercontraction and actin stress fiber formation in the vascular system. ${ }^{22}$ Initially, we investigated whether baicalein acts as a ROCK inhibitor. In our preliminary studies to establish a ROCK kinase assay, we determined the $K_{\mathrm{m}}$ value of ATP $(3 \mu \mathrm{M})$, the $\mathrm{EC}_{50}$ value of $\operatorname{ROCK} 2(0.1 \mu \mathrm{g} / \mathrm{mL})$ and the $\mathrm{EC}_{50}$ value of $\mathrm{S} 6$ ribosomal protein-derived substrate $(1 \mu \mathrm{M})$. Based on these conditions, double reciprocal plots of $1 / v$ versus $1 /[\mathrm{ATP}]$ were made using various concentration of ATP $(0.625-10 \mu \mathrm{M})$, ROCK2 $(0.1 \mu \mathrm{g} /$ $\mathrm{mL})$, and substrate $(1 \mu \mathrm{M})$ in the absence or presence of increasing concentrations of baicalein. The initial rate $v$ was estimated as amount of phospho-substrate transferred during the reaction period ( $\mathrm{nm} / \mathrm{min}$ ). Baicalein was found to behave as an ATP-noncompetitive inhibitor of ROCK2, which suggests that baicalein binds to an allosteric site within ROCK rather than to the ATP binding pocket.

Of the multiple substrates involved in ROCK signaling, MYPT is known to be a major downstream target ${ }^{23)}$ that regulates the interaction between actin and myosin in response to signaling induced by ROCK activation. The phosphorylation of MYPT at Thr696 results in phosphatase inhibition and cytoskeletal reorganization. ${ }^{24)}$ ROCK also phosphorylates Ser19 of MLC2, which directly regulates the assembly of actin stress fiber. ${ }^{25}$ Thus, we investigated whether baicalein regulates the phosphorylations of MYPT1 and MLC2. Immunoblot analysis showed that baicalein suppressed the angiotensin II-induced phosphorylations of MYPT1 and MLC, suggesting that baicalein inhibits the angiotensin II-induced phosphorylations of MYPT1 and MLC2 via ROCK inhibition. In addition, we measured the inhibitory effects of baicalein on the activities of MLCK and PKC $\alpha$ ( $\mathrm{IC}_{50}$ values: $43.4,15.8 \mu \mathrm{M}$, respectively) because MLC2 could be phosphorylated by $\mathrm{MLCK}^{26)}$ or $\mathrm{PKC} \alpha^{27)}$ in cardiomyocytes. These results demonstrate baicalein had more than $>5-10$ fold selectivity towards ROCK 2 and that baicalein can decrease the angiotensin II-induced phosphorylation of MLC2, at least in part, due to its inhibition of MLCK and PKC $\alpha$ beside to ROCK inhibition.

It is known that inappropriate regulation of actin stress fiber formation is directly involved in numerous pathological situations, including cardiovascular disease and cancer. ${ }^{28,29)}$ Although the mechanism responsible for the regulation of stress fiber formation remains to be elucidated, ROCK appears to be a key kinase that directly phosphorylates MLC2 and MYPT and subsequent stress fiber formation. ${ }^{30)}$ In actin stress fiber formation study, baicalein markedly suppressed angiotensin II-induced actin stress fiber formation in H9c2 cells. Furthermore, on increasing the concentration of baicalein, thin central stress fibers were gradually lost, whereas thick peripheral stress fibers remained. These results are typical of ROCK inhibition, ${ }^{31)}$ and imply that baicalein suppresses angiotensin II-induced stress fiber formation by targeting ROCK.

In conclusion, the present results suggest baicalein possesses potent ROCK inhibitory activity, and that ROCK inhibition suppresses actin stress fiber formation by inhibiting the phosphorylations of MLC2 and MYPT. However, further studies are necessary to elucidate the molecular entities directly responsible.

Acknowledgements This study was supported by a Grant of the Bio and Medical Technology Development Program (2011-0019397) of the National Research Foundation (MRF) funded by MEST, and by a Grant of the Technology Innovation Program (10038744) of Korea Evaluation Institute of Industrial Technology (KEIT) funded by MKE, Republic of Korea. 


\section{REFERENCES}

1) Amano M, Fukata Y, Kaibuchi K. Regulation and functions of Rhoassociated kinase. Exp. Cell Res., 261, 44-51 (2000).

2) Kawano Y, Yoshimura T, Kaibuchi K. Smooth muscle contraction by small GTPase Rho. Nagoya J. Med. Sci., 65, 1-8 (2002).

3) $\mathrm{Hu} \mathrm{E}$, Lee $\mathrm{D}$. Rho kinase inhibitors as potential therapeutic agents for cardiovascular diseases. Curr. Opin. Investig. Drugs, 4, 10651075 (2003).

4) Nakagawa O, Fujisawa K, Ishizaki T, Saito $Y$, Nakao K, Narumiya S. ROCK-I and ROCK-II, two isoforms of Rho-associated coiledcoil forming protein serine/threonine kinase in mice. FEBS Lett., 392, 189-193 (1996).

5) Shimokawa H, Rashid M. Development of Rho-kinase inhibitors for cardiovascular medicine. Trends Pharmacol. Sci., 28, 296-302 (2007).

6) Budzyn K, Marley PD, Sobey CG. Targeting Rho and Rho-kinase in the treatment of cardiovascular disease. Trends Pharmacol. Sci., 27, 97-104 (2006)

7) Shimokawa H, Takeshita A. Rho-kinase is an important therapeutic target in cardiovascular medicine. Arterioscler. Thromb. Vasc. Biol. 25, 1767-1775 (2005).

8) Lai A, Frishman WH. Rho-kinase inhibition in the therapy of cardiovascular disease. Cardiol. Rev., 13, 285-292 (2005).

9) Pratt C. Alternative prevention and treatment of cardiovascular disease, part 2. Prim. Care, 37, 339-366 (2010).

10) Tachjian A, Maria V, Jahangir A. Use of herbal products and potential interactions in patients with cardiovascular diseases. J. Am. Coll. Cardiol., 55, 515-525 (2010).

11) Wang CZ, Mehendale SR, Yuan CS. Commonly used antioxidant botanicals: active constituents and their potential role in cardiovascular illness. Am. J. Chin. Med., 35, 543-558 (2007).

12) Shao ZH, Li CQ, Vanden Hoek TL, Becker LB, Schumacker PT, Wu JA, Attele AS, Yuan CS. Extract from Scutellaria baicalensis Georgi attenuates oxidant stress in cardiomyocytes. J. Mol. Cell. Cardiol., 31, 1885-1895 (1999).

13) Chung CP, Park JB, Bae KH. Pharmacological effects of methanolic extract from the root of Scutellaria baicalensis and its flavonoids on human gingival fibroblast. Planta Med., 61, 150-153 (1995).

14) Li HB, Jiang Y, Chen F. Separation methods used for Scutellaria baicalensis active components. J. Chromatogr. B Analyt. Technol. Biomed. Life Sci., 812, 277-290 (2004).

15) Woo AY, Cheng CH, Waye MM. Baicalein protects rat cardiomyocytes from hypoxia/reoxygenation damage via a prooxidant mechanism. Cardiovasc. Res., 65, 244-253 (2005).

16) Shao ZH, Vanden Hoek TL, Qin Y, Becker LB, Schumacker PT, Li CQ, Dey L, Barth E, Halpern H, Rosen GM, Yuan CS. Baicalein attenuates oxidant stress in cardiomyocytes. Am. J. Physiol. Heart Circ. Physiol., 282, H999-H1006 (2002).

17) Lee YM, Cheng PY, Chim LS, Kung CW, Ka SM, Chung MT, Sheu JR. Baicalein, an active component of Scutellaria baicalensis
GEORGI, improves cardiac contractile function in endotoxaemic rats via induction of heme oxygenase-1 and suppression of inflammatory responses. J. Ethnopharmacol., 135, 179-185 (2011).

18) Kong EK, Yu S, Sanderson JE, Chen KB, Huang Y, Yu CM. A novel anti-fibrotic agent, baicalein, for the treatment of myocardial fibrosis in spontaneously hypertensive rats. Eur. J. Pharmacol., 658, 175-181 (2011)

19) Ishizaki $T$, Uehata M, Tamechika I, Keel J, Nonomura K, Maekawa M, Narumiya S. Pharmacological properties of Y-27632, a specific inhibitor of Rho-associated kinases. Mol. Pharmacol., 57, 976-983 (2000).

20) Amano M, Chihara K, Kimura K, Fukata Y, Nakamura N, Matsuura Y, Kaibuchi K. Formation of actin stress fibers and focal adhesions enhanced by Rho-kinase. Science, 275, 1308-1311 (1997).

21) Monceau V, Pasinetti N, Schupp C, Pouzoulet F, Opolon P, Vozenin MC. Modulation of the Rho/ROCK pathway in heart and lung after thorax irradiation reveals targets to improve normal tissue toxicity. Curr. Drug Targets, 11, 1395-1404 (2010).

22) Tsai CC, Liu HF, Hsu KC, Yang JM, Chen C, Liu KK, Hsu TS Chao JI. 7-Chloro-6-piperidin-1-yl-quinoline-5,8-dione (PT-262), a novel ROCK inhibitor blocks cytoskeleton function and cell migration. Biochem. Pharmacol., 81, 856-865 (2011).

23) Terrak M, Kerff F, Langsetmo K, Tao T, Dominguez R. Structural basis of protein phosphatase 1 regulation. Nature, 429, 780-784 (2004).

24) Birukova AA, Smurova K, Birukov KG, Usatyuk P, Liu F, Kaibuchi K, Ricks-Cord A, Natarajan V, Alieva I, Garcia JG, Verin AD. Microtubule disassembly induces cytoskeletal remodeling and lung vascular barrier dysfunction: role of Rho-dependent mechanisms. $J$ Cell. Physiol., 201, 55-70 (2004).

25) Totsukawa G, Yamakita Y, Yamashiro S, Hartshorne DJ, Sasaki Y, Matsumura F. Distinct roles of ROCK (Rho-kinase) and MLCK in spatial regulation of MLC phosphorylation for assembly of stress fibers and focal adhesions in $3 \mathrm{~T} 3$ fibroblasts. J. Cell Biol., 150, 797-806 (2000).

26) Chan JY, Takeda M, Briggs LE, Graham ML, Lu JT, Horikoshi N, Weinberg EO, Aoki H, Sato N, Chien KR, Kasahara H. Identification of cardiac-specific myosin light chain kinase. Circ. Res., 102, 571-580 (2008)

27) Pucéat $M$, Vassort G. Signalling by protein kinase $C$ isoforms in the heart. Mol. Cell. Biochem., 157, 65-72 (1996).

28) Chaponnier C, Gabbiani G. Pathological situations characterized by altered actin isoform expression. J. Pathol., 204, 386-395 (2004).

29) Jin L. The actin associated protein palladin in smooth muscle and in the development of diseases of the cardiovasculature and in cancer. J. Muscle Res. Cell Motil., 32, 7-17 (2011).

30) Pellegrin S, Mellor H. Actin stress fibres. J. Cell Sci., 120, 34913499 (2007).

31) Katoh K, Kano Y, Amano M, Kaibuchi K, Fujiwara K. Stress fiber organization regulated by MLCK and Rho-kinase in cultured human fibroblasts. Am. J. Physiol. Cell Physiol., 280, C1669-C1679 (2001). 